\title{
Optical Coherence Tomography Angiography of Choroidal Neovascularisation
}

\author{
Lorenzo Iuliano \\ Ophthalmology Department, Vita-Salute University, San Raffaele Scientific Institute, Milan, Italy
}

DOI: http://doi.org/10.17925/EOR.2016.10.01.29

\begin{abstract}
Optical coherence tomography angiography (OCTA) is a new promising technique able to provide a rapid, dyeless and non-invasive three-dimensional reconstruction of perfused vessels of retina and choroid. OCTA has been recently added to the diagnostic tools of retinal experts, and its role is still being explored in different retinal diseases. Chorodial neovascularisations (CNVs) are among the most significant disorders where OCTA is enhancing our diagnostic and classification skills, since it demonstrates an excellent capacity to identify and characterise each specific subtype of CNV.
\end{abstract}

\section{Keywords}

Choroidal neovascularisation, optical coherence tomography angiography, polypoidal choroidal vasculopathy, retinal angiomatous proliferation, capillary plexus

\begin{abstract}
Disclosure: Lorenzo Iuliano did not receive any financial or material support for the preparation of this manuscript. He has no financial or proprietary interest related to the work. This article is a short opinion piece and has not been submitted to external peer reviewers.

Open Access: This article is published under the Creative Commons Attribution Nonco mmercial License, which permits any non-commercial use, distribution, adaptation and reproduction provided the original author(s) and source are given appropriate credit.

Received: 10 June 2016 Published Online: 4 August 2016 Citation: European Ophthalmic Review, 2016;10(1):29-30

Correspondence: Lorenzo Iuliano, Department of Ophthalmology, Vita-Salute University, San Raffaele Scientific Institute, Via Olgettina, 60, 20132 Milan, Italy.

E: iuliano.lorenzo@hsr.it
\end{abstract}

Optical coherence tomography angiography (OCTA) is a relatively new diagnostic technique that is able to provide a rapid, dyeless and noninvasive three-dimensional reconstruction of perfused vessels of retina and choroid.

The fundamental concept of OCTA is that in a static eye the sole moving structure is blood, flowing through retinal vessels: indeed, erythrocyte movement is the contrast medium. The basis of the technique is to perform repeated scans in the same area, and extract the changes in the resultant images. The signal is generated by way of a mathematic process (decorrelation algorithm) that produces clear differentiation between blood flow and static tissue. Image contrast is produced by the difference between the changes (moving cells) and the static surrounding tissue.

Some artifacts may arise from physiological microsaccades and eye throbbing. In order to reduce these artifacts, a sequence of B-scans in the exact same retinal location must be acquired to detect flow. In addition, active eye tracking, using simultaneous acquisition of fundus and OCTA images, is employed to reduce errors.

Each commercially available device includes a specific algorithm (e.g., SSADA by Optovue Angiovue system, OCTARA by Topcon, Angioplex by Zeiss), with exclusive advantages and disadvantages. The standard automatic segmentation of the retinal capillary bed distinguishes two intraretinal vascular layers: the superficial capillary plexus (SCP), allocated in the ganglion cell layer, and the deep capillary plexus (DCP), in the inner nuclear layer. The third vascular bed automatically segmented is the choriocapillary (CC), immediately under the Bruch membrane. In a healthy individual, the tissue space between the DCP and the CC is optically empty, and is therefore named avascular zone. A manual segmentation is also possible, giving the clinician the possibility to explore selected areas of interest.

Choroidal neovascular (CNV) are new blood vessels that grow beneath the retina and disrupt vision. These blood vessels start to grow in the choroid and may break through the retinal pigment epithelium (RPE), leaking blood and fluid that damages the photoreceptors. CNVs are classified into three types. Type $1 \mathrm{NV}$ originates from the CC and is localised under the RPE. Type 2 also originates from the CC, but extends through the RPE and invades the subretinal space. Type 3 NV essentially includes two previously described lesions: retinal angiomatous proliferation (RAP) and chorioretinal anastomosis. RAP consists of focal NV proliferation from the DCP that extends into the subretinal space. It may eventually communicate with a type $1 \mathrm{CNV}$. Chorioretinal anastomosis is defined as an intraretinal extension of type $1 \mathrm{NV}$.

Type $1 \mathrm{NV}$ is the most common NV subtype of age-related macular degeneration (AMD). OCTA is able to provide more precise identification and classification of type $1 \mathrm{NV}$ than fluorescein angiography (FA) and indocyanine green angiography (ICGA). Type $1 \mathrm{CNV}$ derives from the CC and is located under the RPE. FA is able to explore the SCP, but poorly visualises the DCP and the CC. Pigmentary epithelium detachment (PED), for example, display pooling or stippled fluorescence, but the identification of the causative NV is sometimes challenging, and occasionally improved with ICGA. In FA and ICGA the presence of type 
$1 \mathrm{CNV}$ is deduced by the presence of pooling within PED and/or the presence of hot spots. On the contrary, OCTA directly shows the vessels themselves, and allows the investigator to accurately diagnose and classify the NV complex. ${ }^{2}$

Type 2 NV typically complicates high myopia and inflammatory retinopathies, but may also be present in AMD. Type 2 CNV discloses very typical OCTA patterns. NV membrane appears as either a medusashaped complex or a glomerulus-shaped lesion, typically located in the outer retina and CC layer. The medusa-shaped has well-defined oval shape, produced by a very dense high-flow network (highly active NV at presentation). The glomerulus-shaped lesion is a round, well defined and dense tangle of small new vessels. Both lesions may also be found on segmentation performed at the level of the CC.

In the CC projection, the peripheral margins of the lesion display as a dark ring, and one or more central feeder vessels extending deeply in the choroidal layers are usually visible. The clinician may easily diagnosis type $2 \mathrm{NV}$ using OCTA, especially in retinal areas where normally vessels are absent, as in the subretinal space. After long-term treatment, the NV lesion becomes fibrotic and its OCTA features change. ${ }^{3}$

Type 3 NV appears as a retina-retinal anastomosis emerging from the DCP, originating a high-flow and tuft-shaped NV lesion. It could be segmented in the outer retinal layers, and in late-stage disease it finally crosses the subretinal RPE space. In the CC segmentation, a small clewlike lesion corresponds to the above-mentioned tuft-shaped network. In some cases, this glomerular lesion is connected with the choroid though a small-caliber vessel. ${ }^{4}$

The CNV subtypes are usually classified using FA and/or ICGA (classic, prevalently classic, minimally classic, occult), but OCTA easily depicts the exact anatomy and the distribution of the vascular networks, both above and below the RPE. This aspect is of paramount importance, particularly in the case of mixed subtypes of CNVs. ${ }^{5}$

Polypoidal choroidal vasculopathy (PCV) typically presents as hypoflow round lesion on OCTA. Signal absence is not indicating blood flow lack, but rather that blood flow is not within the device detection threshold. This could be due to either increased or decreased flow in the polyps and subsequent nonvisualisation of the vascular structure. It has been hypothesised that the apparent signal absence in PCV could be due either to the turbulent blood flow inside of the polyps, or to the fact that blood circulates only at the aneurism periphery. ${ }^{6}$

Another critical aspect of this new imaging method is to describe the changes of both CNV natural history and after treatment. In early phases of presentation, NV appears as a tangled web of fine vessels, as a round tuft of small-caliber capillaries without dilated core feeder vessels. Chronic lesions demonstrate a distinctly different morphology, showing a highly organised vascular complex with vessels branching from a core trunk of multiple large, dilated feeder vessels. It has been proposed that these central trunks are more resistant to anti-VEGF treatment, since overlying pericytes protect their endothelial cells. Indeed, after each anti-VEGF injection a pruning of smaller vessels is immediately visible (after 24 hours), which intensify for 6-12 days. Reopening of new sprouting vessels is evident 20-50 days later, in case of CNV reactivation. Pruning the small peripheral branches seems to strengthen and increased the flow of the vascular trunk. This phenomenon, on OCTA, is known as 'arterialisation' of the main vascular trunk. ${ }^{2,7}$

OCTA recently also raised interest in the scientific community on fibrosis and on low-activity (quiescent) CNVs. In almost all of fibrotic CNVs, a high-flow network was found inside the scar. Different vascular patterns have been also described (pruned vascular tree, tangled network, vascular loop). OCTA of subretinal fibrosis recently demonstrated a perfused and abnormal vascular network, as well as collateral architectural changes in the outer retina and in the CC. ${ }^{8}$

One of the crucial aspects of assessing OCTA validity is to compare its diagnostic ability to gold standard techniques, such as FA and/or ICGA. One could also ask whether this new imaging method may replace in the future the traditional invasive methods of angiography. Actual studies report that OCTA specificity to detect CNV is $67.6 \%$, and sensitivity is $86.5 \%$. This data place now OCTA as a good strategy for diagnosis rather than for follow-up, but further studies are required to better assess the specific characteristics of CNV throughout treatment.
1. Chalam KV, Sambhav K, Optical coherence tomography angiography in retinal diseases, $J$ Ophthalmic Vis Res, 2016:11:84-92.

2. lafe NA, Phasukkijwatana N, Sarraf D, Optical Coherence Tomography Angiography of Type 1 Neovascularization in Age-Related Macular Degeneration, Dev Ophthalmol, 2016;56:45-51

3. Souied EH, El Ameen A, Semoun O, et al., Optical Coherence Tomography Angiography of Type 2 Neovascularization in Age-Related Macular Degeneration, Dev Ophthalmol, 2016;56:52-6.
4. Querques G, Miere A, Souied EH, Optical Coherence Tomography Angiography Features of Type 3 Neovascularization in Age-Related Macular Degeneration, Neovascularization in Age-Related

5. Liang MC, Witkin AJ, Optical Coherence Tomography Angiography of Mixed Neovascularizations in Age-Relate Macular Degeneration, Dev Ophthalmol, 2016;56:62-70.

6. Srour M, Querques G, Souied EH, Optical Coherence Tomography Angiography of Idiopathic Polypoidal Choroidal Vasculopathy, Dev Ophthalmol, 2016;56:71-6.

7. Lumbroso B, Rispoli M, Savastano MC, et al., Optica
Coherence Tomography Angiography Study of Choroida Neovascularization Early Response after Treatment, Dev Ophthalmol, 2016;56:77-85.

8. Souied EH, Miere A, Cohen SY, et al., Optical Coherence Tomography Angiography of Fibrosis in Age-Related Macular Degeneration, Dev Ophthalmol, 2016;56:86-90.

9. Gong J, Yu S, Gong Y, et al., The Diagnostic Accuracy of optical Coherence Tomography Angiography for Neovascular Age-Related Macular Degeneration: A Comparison with Fundus Fluorescein Angiography, J Ophthalmol, 2016;2016:7521478. 